\begin{tabular}{|c|l|}
\hline Title & Quantitative magnetic field imaging by polarized pul sed neutrons at J-PA RC \\
\hline Author(s) & $\begin{array}{l}\text { Shinohara, T.; Sakai, K.; Ohi, M.; Kai, T.; Harada, M.; Oikawa, K.; Maekawa, F.; Suzuki, J.; O ku, T.; T akata, S.; } \\
\text { A izawa, K.; A rai, M.; Kiyanagi, Y. }\end{array}$ \\
\hline Citation & $\begin{array}{l}\text { Nuclear Instruments and Methods in Physics Research Section A : A ccelerators, Spectrometers, Detectors and } \\
\text { A ssociated Equipment, 651(1), 121-125 } \\
\text { https:/doi.org/10.1016/.nima.2011.01.099 }\end{array}$ \\
\hline Issue Date & 2011-09-21 \\
\hline Doc URL & http://hdl.handle.net/2115/47381 \\
\hline Type & article (author version) \\
\hline File Information & NIMPRA651-1_121-125.pdf \\
\hline
\end{tabular}

Instructions for use 


\title{
QUANTITATIVE MAGNETIC FIELD IMAGING BY POLARIZED PULSED NEUTRONS AT J-PARC
}

\author{
T. Shinohara ${ }^{\text {a, }}$, K. Sakai ${ }^{a}$, M. Ohi ${ }^{a}$, T. Kai ${ }^{a}$, M. Harada ${ }^{a}$, K. Oikawa ${ }^{a}$, F. \\ Maekawa $^{a}$, J. Suzuki ${ }^{a}$, T. Oku ${ }^{a}$, S. Takata ${ }^{a}$, K. Aizawa ${ }^{a}$, M. Arai $^{a}$, and Y. \\ Kiyanagi $^{b}$
}

a J-PARC Center, Japan Atomic Energy Agency, 2-4 Shirakata-shirane, Tokai, Ibaraki 319-1195, Japan;

${ }^{\mathrm{b}}$ Faculty of Engineering, Hokkaido University, Kita-13, Nishi-8, Kita-ku, Sapporo, Hokkaido 060-8628, Japan.

${ }^{*}$ Corresponding author: Dr. Takenao Shinohara

takenao.shinohara@j-parc.jp

2-4 Shirakata-shirane, Tokai, Ibaraki 319-1195, Japan.

Tel: +81-29-284-3753; Fax: +81-29-284-3889;

Abstract: One of the most interesting applications of neutron imagining is that it enables the observation of magnetic field. Because the interaction between the neutron spin and the magnetic field depends on the neutron energy, it is important to study the neutron energy dependence of magnetic field imaging to quantitatively treat the characteristics of the magnetic field. In this study, we have performed a polarized pulsed neutron imaging experiment using a timeof-flight method at J-PARC and have analyzed the energy dependence of the obtained polarization image. We have also quantitatively analyzed the strength and the direction of the magnetic field relative to the quantized axis inside a solenoid coil.

Keywords: Polarized neutrons; Pulsed neutron imaging; Time-of-flight method; Magnetic fields. 


\section{Introduction}

Neutron imaging is one of the fundamental techniques that is used to observe the internal structures of objects and to visualize them in real space. Recently, an energy-selective neutron imaging technique has been developed $[1,2]$. The advantage of this technique is that it enables not only the enhancement of intensity contrast by the selection of neutron energy but also the acquisition of neutron transmission spectra with spatial resolution. Consequently, this technique enables to extract the distribution of various types of physical or chemical information about objects quantitatively, for example, their crystallographic information or their elemental composition. Therefore, energy-selective neutron imaging can be regarded as a technique that can be used to measure physical quantities and visualize them based on the neutron transmission.

On the other hand, because a neutron has a magnetic moment, it is possible to observe magnetic fields inside materials or a space by neutron spin analysis [3-8]. Because the motion of a neutron in a magnetic field intrinsically depends on its spin polarity and energy, a polarized monochromatic beam is required for magnetic field imaging. We briefly describe the principle of magnetic field imaging by considering the interaction between a neutron and a magnetic field. The equation of motion of a neutron spin passing through a magnetic field can be expressed as follows:

$$
\frac{\mathrm{d}}{\mathrm{d} t} \boldsymbol{\sigma}(t)=\gamma \boldsymbol{\sigma}(t) \times \boldsymbol{B}(t)
$$

where $\sigma(t)$ is the unit vector parallel to the neutron spin, $\gamma$ is the gyromagnetic ratio of the neutron, and $\boldsymbol{B}(t)[T]$ is the external magnetic field. It is seen that the neutron spin experiences a Larmor precession in a given magnetic field. The precession angle $\varphi$ can be expressed in terms of the product of the precession frequency $\omega_{\llcorner}$and the residence time $t$ in a magnetic field $B=$ $|\boldsymbol{B}(t)|$; it can also be expressed in terms of the neutron velocity $v[\mathrm{~m} / \mathrm{s}]$ and the wavelength $\lambda[\AA]$ as follows:

$$
\varphi=\omega_{L} t=\frac{\gamma}{v} \int_{\text {path }} B \mathrm{~d} s=\frac{\gamma \lambda}{3956} \int_{\text {path }} B \mathrm{~d} s
$$


In the above equation, the rotation angle $\varphi$ denotes the cumulated magnetic field along the beam path when the incident polarized neutron beam is monochromatic. It is found that the spatial distribution of polarization of the transmitted neutron, which is in fact the projection of $\varphi$, is responsible for the formation of an image related to the magnetic field. Furthermore, the measurements of $\varphi$ as a function of neutron velocity or wavelength make it possible to evaluate the path integral of the magnetic field in which neutrons transverse. Accordingly, the analysis of the wavelength dependence of neutron polarization image will enable us to investigate details about the magnetic field characteristics with the spatial resolution. On the basis of this concept, we explore the use of a neutron imaging technique with energy selection and polarization analysis to achieve quantitative magnetic field imaging.

Pulsed neutrons are intrinsically suitable to study the neutron energydependent phenomena, because they are monochromatic at each instant and their energy can be measured precisely using a time-of-flight (TOF) method. Further, because wide energy range neutrons are available simultaneously, i.e., from epithermal neutrons or higher energy neutrons to cold neutrons, highly efficient energy dependent measurements can be performed using pulsed neutrons. In Japan, a new MW-class pulsed neutron source of the Japan Spallation Neutron Source (JSNS), was constructed in the Materials and Life Science Facility (MLF) of the Japan Proton Accelerator Research Complex (J-PARC) [9]. At present, we are developing energy-resolved polarized pulsed neutron imaging techniques to quantitatively analyze the characteristics of the magnetic field, particularly its strength and direction, at J-PARC.

In this work, we performed the experiments of the polarized pulsed neutron imaging using a polarization analysis setup in transmission mode combined with the TOF analysis. We determined the strength and direction of the magnetic field from the results of the energy-dependent polarization analysis.

\section{Experimental Procedures}


Magnetic field imaging experiments using a polarized pulsed neutron beam were performed at the beam line of BL10 "NOBORU", which is installed in the first experimental hall of MLF at J-PARC. Detailed information about this beam line has been provided elsewhere [10]. Fig. 1 shows a schematic illustration of the experimental setup, which mainly comprises two polarizing devices (a polarizer and an analyzer), a spin flipper, a two-dimensional neutron detector, and a sample. The pulsed neutrons provided from the decoupled hydrogen moderator of the JSNS were polarized by a transmission-type polarizer using stacked bent magnetic super-mirrors [11], and their polarization was analyzed using an analyzer, which is similar device as the polarizer. The neutrons reflected by the analyzer were eliminated using a soller collimator placed behind the analyzer. A vertically oriented magnetic field was applied to both the polarizer and the analyzer using permanent magnets in order to magnetize the magnetic super-mirrors, and its stray fields were used as the guide fields to prevent depolarization during the passage of the polarized neutron beam. The neutron spin polarity of the incident beam was reversed using an adiabatic fast passage spin flipper placed behind the polarizer [12]. The analyzed neutrons transmitted through the collimator were detected by a two-dimensional neutron detector, which was composed of a position-sensitive photomultiplier tube with a $\mathrm{ZnS}(\mathrm{Ag}) /{ }^{6} \mathrm{LiF}$ scintillator on the surface (this detector is called RPMT), and information about the position and the flight time of the neutron was recorded for each event. The RPMT had a spatial resolution of approximately $1 \mathrm{~mm}$ and a time resolution of $10 \mu \mathrm{s}$ [13]. The beam had a height of $20 \mathrm{~mm}$ and a width of $10 \mathrm{~mm}$. These dimensions were defined using the $\mathrm{B}_{4} \mathrm{C}$ slits installed at a distance of $7.05 \mathrm{~m}$ and $12.75 \mathrm{~m}$ from the neutron source, so as to fit the aperture size of the polarization devices. Because the objective of this study was to evaluate the characteristics of the magnetic field by analyzing the energy dependence of the polarization image using pulsed neutrons, a simple magnetic object was preferred as the sample. Therefore, to meet this objective, in this study, we prepared a small solenoid coil with a diameter of $5 \mathrm{~mm}$ and a length of approximately $30 \mathrm{~mm}$. This coil was mounted at a distance of $13.88 \mathrm{~m}$ from the source, which is exactly midway between the spin flipper and the analyzer. 
The exposure time for one measurement was approximately $30 \mathrm{~min}$, which corresponded to 50,000 pulses at the JSNS.

Fig. 1. Schematic illustration of the experimental setup. The coil and the detector were placed at a distance of $13.88 \mathrm{~m}$ and $14.39 \mathrm{~m}$ from the neutron source, respectively.

Polarization along the quantized axis was determined from the transmitted intensities of the spin-up neutrons and the spin-down neutrons. In practical experiments, we could not measure the exact numbers of both the spin-up neutrons and the spin-down neutrons owing to the ambiguity in the efficiencies of the polarizer, analyzer, and flipper. Alternatively, we performed measurements in which the spin flipper was switched on $\left(\mathrm{I}_{\mathrm{on}}\right)$ and off $\left(\mathrm{I}_{\mathrm{off}}\right)$ under all experimental conditions. From these measurements, we obtained $P_{i}=\left(I_{\text {off }}-I_{\text {on }}\right) /\left(I_{\text {off }}+I_{\text {on }}\right)$. To cancel the effects of the polarizing efficiencies, $P_{i}$ was normalized on the basis of the results obtained without a sample $\mathrm{P}_{0}$. Further, in this calculation, the attenuation of the neutron beam owing to the absorption by the sample was corrected automatically [14]. Then, we obtained the energy dependence, namely the wavelength dependence, of polarization by performing the TOF analysis of pulsed neutrons.

\section{Results and Discussion}

First, we present the results of the degree of polarization of the incident neutron beam $\mathrm{P}_{0}$ obtained by the measurements performed without a sample. The wavelength dependence of polarization showed that neutrons with a wavelength longer than $3 \AA$ could be polarized with our experimental system and that the degree of polarization reached a maximum value of $96 \%$ at a wavelength of $6 \AA$. The obtained two-dimensional polarization image showed that the spatial distribution of the polarization of the incident beam was confirmed to be almost homogeneous.

Fig. 2 shows the images of polarization distribution obtained at a wavelength of $6.3 \AA$, when the sample coil was placed in the beam path. This coil was arranged to create a magnetic field perpendicular to the direction of 
the guide field (Fig. 2 (c)), because this field arrangement causes the largest change in the degree of polarization owing to neutron spin precession. By comparing the images shown in this figure, a change in the degree of polarization at the position of the sample coil is apparently observed when an electric current is applied to the coil.

Images of polarization distribution and their profiles along the vertical axis obtained for various neutron wavelengths are shown in Fig. 3. This figure shows that polarization at the position of the coil decreases at first and then recovers with an increase in the wavelength. This oscillation behavior of polarization is indicative of the neutron spin rotation around the magnetic field in the sample coil. Therefore, in this study, a change in the polarization image depending on the neutron wavelength was successfully observed using polarized pulsed neutrons. It is worth mentioning that the wavelengthdependent polarization images could be obtained by performing only a couple of measurements, in which the spin flipper was turned on and off, using the TOF method with pulsed neutrons.

Fig. 2. Images of polarization distribution obtained at a wavelength of $6.3 \AA$. (a) Sample coil without applied current and (b) with an applied current of $1.8 \mathrm{~A}$. The channel width is $0.5 \mathrm{~mm}$. (c) Arrangement of magnetic field.

Fig. 3. (a) Polarization distribution images obtained for several wavelengths and (b) corresponding polarization profiles along the vertical axis. White dotted lines in (a) indicate the lines along which polarization profiles shown in (b) were obtained.

Fig. 4 shows the wavelength dependences of the normalized polarization at the center of the coil for various applied current conditions. In this figure, a sinusoidal oscillation, which is theoretically expected, of polarization against wavelength is clearly observed. It is also observed that the periods of polarization oscillation shorten with an increase in the applied current. According to the precession angle $\varphi$ described by eq. (2), the frequency of polarization oscillation is proportional to the path integrals of the 
magnetic field. Hence, the shortening of the oscillation period relative to the applied current can be regarded as an increase in the magnetic field strength inside the sample coil. By fitting these data with cosine curves, we evaluated the oscillation frequency for each applied current condition. Assuming that the magnetic field $B$ is uniform inside the coil and that the path length is equal to the inner diameter of the coil, we can easily calculate the precession frequency $\omega_{L}$ from the oscillation frequency. As a result, the strength of $B$ was estimated to be 2.2 [mT/A], which agrees well with the designed value of the coil. Therefore, by means of these analyses, we could quantify the magnetic field strength from the results of wavelength-dependent magnetic imaging experiments.

Fig. 4. Wavelength dependence of normalized polarization obtained at various applied current conditions. The lines indicate the results of fitting of wavelength-dependent polarization with a sinusoidal function.

Next, the configuration of the sample coil was changed by rotating it slightly in the plane perpendicular to the direction of the neutron beam, and measurements were performed with an applied current of $2.5 \mathrm{~A}$. The typical image of polarization distribution and the wavelength dependence of the normalized polarization calculated at the center of the coil with an area of 2.5 $\times 2.5 \mathrm{~mm}^{2}$ are shown in Fig. 5. While we can distinguish the position of the sample coil from the polarization image shown in this figure, we cannot determine the direction the magnetic field only from this image. Owing to the precession process around the magnetic field tilted from the quantized axis of the incident neutron spin, a shift in the axis, around which polarization oscillates, will occur according to the tilt angle. In fact, this shift in the axis was confirmed by the wavelength dependence of polarization, as shown in Fig. 5(b). By fitting the results of wavelength-dependent polarization with the sinusoidal function in a manner similar to that used in the previous analysis, we evaluated the amount of shift observed in the polarization oscillation to be $0.38 \pm 0.02$. From this value, we calculated the direction of the magnetic field relative to the quantized axis to be $49 \pm 3^{\circ}$. Because the inclination of the 
coil's central axis from the vertical axis was estimated to be $47.2^{\circ}$ from the polarization image, the magnetic field was confirmed to align with the center axis of the coil.

Fig. 5. (a) Polarization distribution image and (b) wavelength dependence of normalized polarization. The polarization shown in (b) was obtained by averaging the neutron intensity of the central $2.5 \times 2.5 \mathrm{~mm}^{2}$ area of the coil. The line in (b) indicates the result of fitting of wavelength-dependent polarization with a sinusoidal function.

On the basis of the simple analysis of wavelength-dependent polarization imaging data using a sinusoidal function, we could successfully quantify both the magnetic field strength and the direction relative to the incident neutron spin of the sample coil. By performing this analysis for each pixel of the image, we can reconstruct the intensity or the direction map of the magnetic field. Results of this analysis indicate that polarized pulsed neutrons are suitable for the quantitative magnetic field imaging experiment. However, this simple oscillation analysis cannot determine each element of the threedimensional magnetic field vector. Therefore, it becomes indispensable to use a three-dimensional polarization analysis technique similar to a neutron polarimetry technique $[15,16]$ for advanced polarized pulsed neutron imaging. Moreover, the application of a depolarization analysis method [14, 17], which is commonly used for the study of magnetic properties inside materials, such as their magnetic domain structures or magnetization processes, to polarized pulsed neutron imaging will enable us to study more complicated magnetic systems.

\section{Conclusion}

In this study, we have performed magnetic field imaging experiments using polarized pulsed neutrons at J-PARC, and we have successfully observed the magnetic field inside a solenoid coil and quantified the strength of the magnetic field by analyzing the wavelength dependence of polarization. Moreover, we have evaluated the direction of the magnetic field relative to the 
quantized axis from the oscillation profiles of neutron polarization as a function of the wavelength. These results indicate that it is possible to quantitatively evaluate both the strength and the direction of the magnetic field with spatial resolution by analyzing the wavelength dependence of polarization using polarized pulsed neutron imaging.

\section{Acknowledgements}

The authors would like to thank Dr. M. Matsubayashi of JAEA for his contribution to our pulsed neutron imaging project. This work was partially supported by a Grant-in-Aid for Scientific Research (C) from the Japan Society for the Promotion of Science (No. 22604009). 


\section{References}

[1] Y. Kiyanagi, H. Sato, O. Takada, N. Ayukawa and T. Kamiyama, AccApp09 Proc. AP/AM-07.

[2] H. Sato, T. Kamiyama and Y. Kiyanagi, Nucl. Instr. and Meth. A 605 (2009) 36-39.

[3] N. Kardjilov, I. Manke, M. Strobl, A. Hilger, W. Treimer, M. Meissner, T.

Krist and J. Banhart, Nat. Phys. 4 (2008) 399-403.

[4] M. Schulz, A. Neubauer, M. Mühlbauer, E. Calzada, B. Schillinger, C.

Pfleiderer and P. Böni, J. Phys: Conference Series 200 (2010) 112009.

[5] M. Schlenker, W. Bauspiess, W. Graeff, U. Bonse and H. Rauch, J. Magn. Magn. Mater. 15-18 (1980) 1507-1509.

[6] F. M. Piegsa, B. van den Brandt, P. Hautle, J. Kohlbrecher and J. A.

Konter, Phys. Rev. Lett. 102 (2009) 145501.

[7] M. Strobl, W. Treimer, P. Walter, S. Keil and I. Manke, Appl. Phys. Lett. 91 (2007) 254104.

[8] C. Grünzweig, C. David, O. Bunk, M. Dierolf, G. Frei, G. Kühne, J.

Kohlbrecher, R. Schäfer, P. Lejcek, H. M. R. Rønnow and F. Pfeiffer, Phys.

Rev. Lett. 101 (2008) 025504.

[9] http://j-parc.jp/MatLife/en/instrumentation/ns.html; http://j-

parc.jp/MatLife/en/instrumentation/ns3.html.

[10] K. Oikawa, F. Maekawa, M. Harada, T. Kai, S. Meigo, Y. Kasugai, M. Ooi, K. Sakai, M. Teshigawara, S. Hasegawa, M. Futakawa, Y. Ikeda and N. Watanabe, Nucl. Instr. and Meth. A 589 (2008) 310-317; F. Maekawa, K.

Oikawa, M. Harada, T. Kai, S. Meigo, Y. Kasugai, M. Ooi, K. Sakai, M.

Teshigawara, S. Hasegawa, Y. Ikeda and N. Watanabe, Nucl. Instr. and Meth. A 600 (2009) 335-337.

[11] T. Krist, S. J. Kennedy, T. J. Hicks and F. Mezei, Phys. B 241-243 (1998) 82-85; T. Krist, Nucl. Instr. and Meth. A 529 (2004) 50-53.

[12] H. Weinfurter and G. Badurek, Nucl. Instr. and Meth. A 275 (1989) 233-

238; W. Faber, B. Bader, P. Heitjans and A. Schirmer, Nucl. Instr. and Meth. A 294 (1990) 241-244. 
[13] K. Hirota, T. Shinohara, K. Ikeda, K. Mishima, T. Adachi, T. Morishima, S.

Satoh, T. Oku, S. Yamada, H. Sasao, J. Suzuki and H. M. Shimizu, Phys.

Chem. Chem. Phys. 7 (2005) 1836-1838.

[14] S. Mitsuda and Y. Endoh, J. Phys. Soc. Jpn. 54 (1985) 1570-1580.

[15] F. Tasset, Phys. B 156\&157 (1989) 627-630.

[16] M. Janoschek, S. Klimko, R. Gähler, B. Roessli and P. Böni, Phys. B 397 (2007) 125-130.

[17] M. T. Rektveldt, Z. Phys. 259 (1973) 391-410. 


\section{CAPTION LIST (On separate page)}

Fig. 1. Schematic illustration of the experimental setup. The coil and the detector were placed at a distance of $13.88 \mathrm{~m}$ and $14.39 \mathrm{~m}$ from the neutron source, respectively.

Fig. 2. Images of polarization distribution obtained at a wavelength of $6.3 \AA$. (a) Sample coil without applied current and (b) with an applied current of $1.8 \mathrm{~A}$. The channel width is $0.5 \mathrm{~mm}$. (c) Arrangement of magnetic field.

Fig. 3. (a) Polarization distribution images obtained for several wavelengths and (b) corresponding polarization profiles along the vertical axis. White dotted lines in (a) indicate the lines along which polarization profiles shown in (b) were obtained.

Fig. 4. Wavelength dependence of normalized polarization obtained at various applied current conditions. The lines indicate the results of fitting of wavelength-dependent polarization with a sinusoidal function.

Fig. 5. (a) Polarization distribution image and (b) wavelength dependence of normalized polarization. The polarization shown in (b) was obtained by averaging the neutron intensity of the central $2.5 \times 2.5 \mathrm{~mm}^{2}$ area of the coil. The line in (b) indicates the result of fitting of wavelength-dependent polarization with a sinusoidal function. 
Figure-1

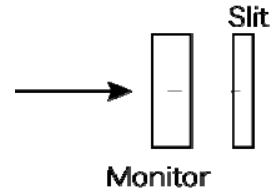

$12.75 \mathrm{~m}$

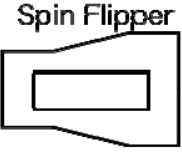

Polarizer

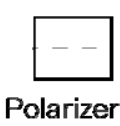

Sample

(Solenoid)

O<smiles>C=[Tl]</smiles>

$13.88 \mathrm{~m}$

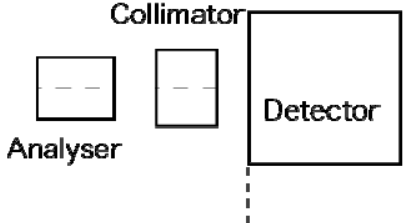

$14.39 \mathrm{~m}$ 
Figure-2
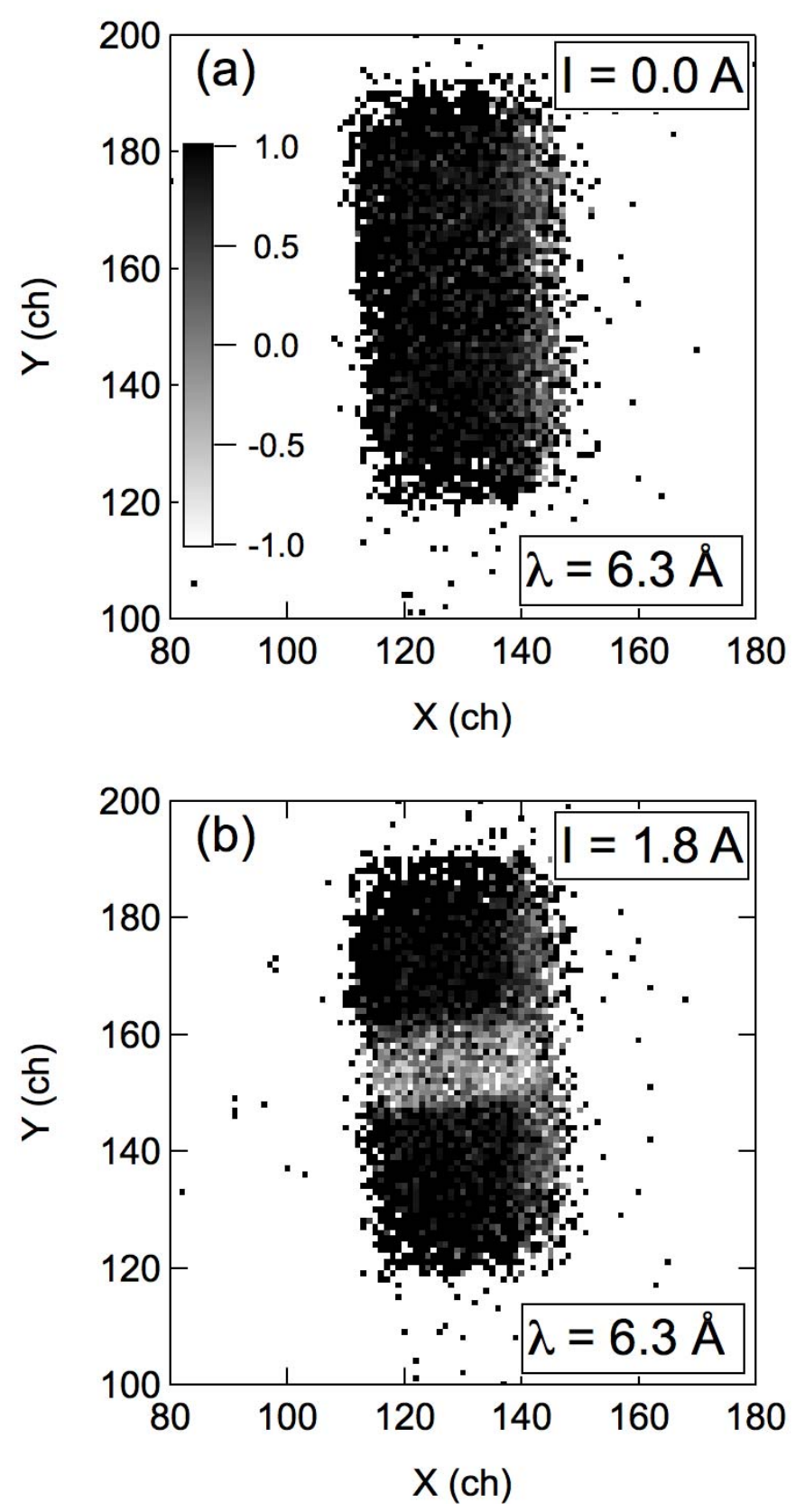

(c)

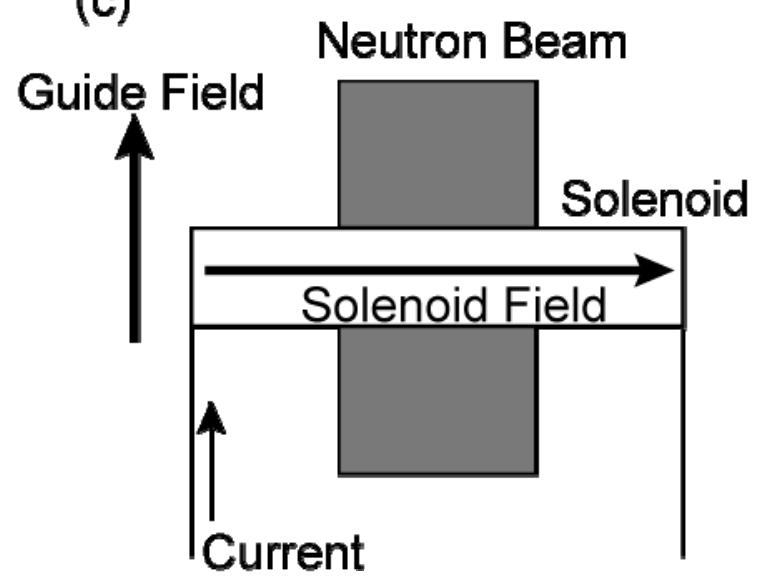




\section{Figure-3}

(a)

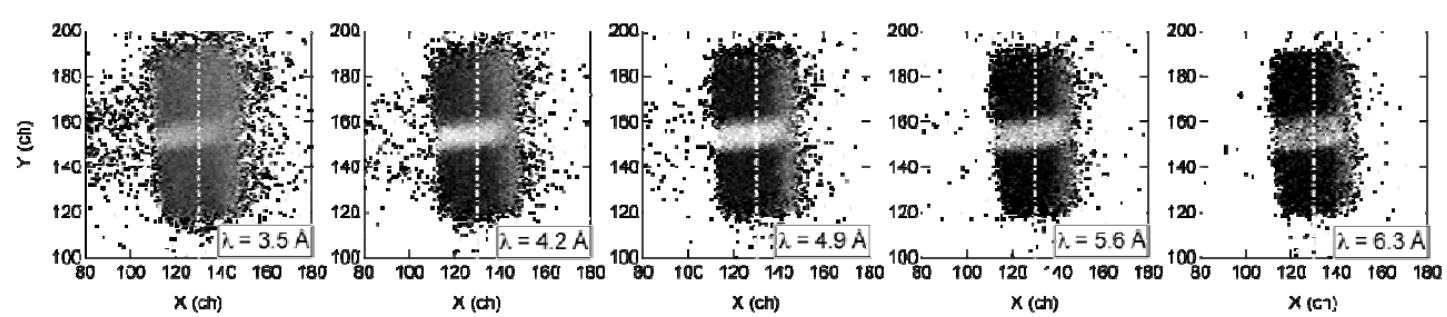

(b)

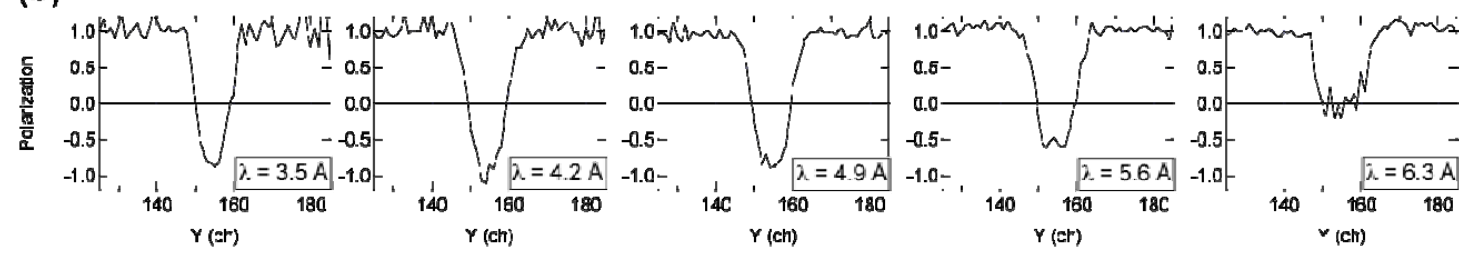


Figure-4

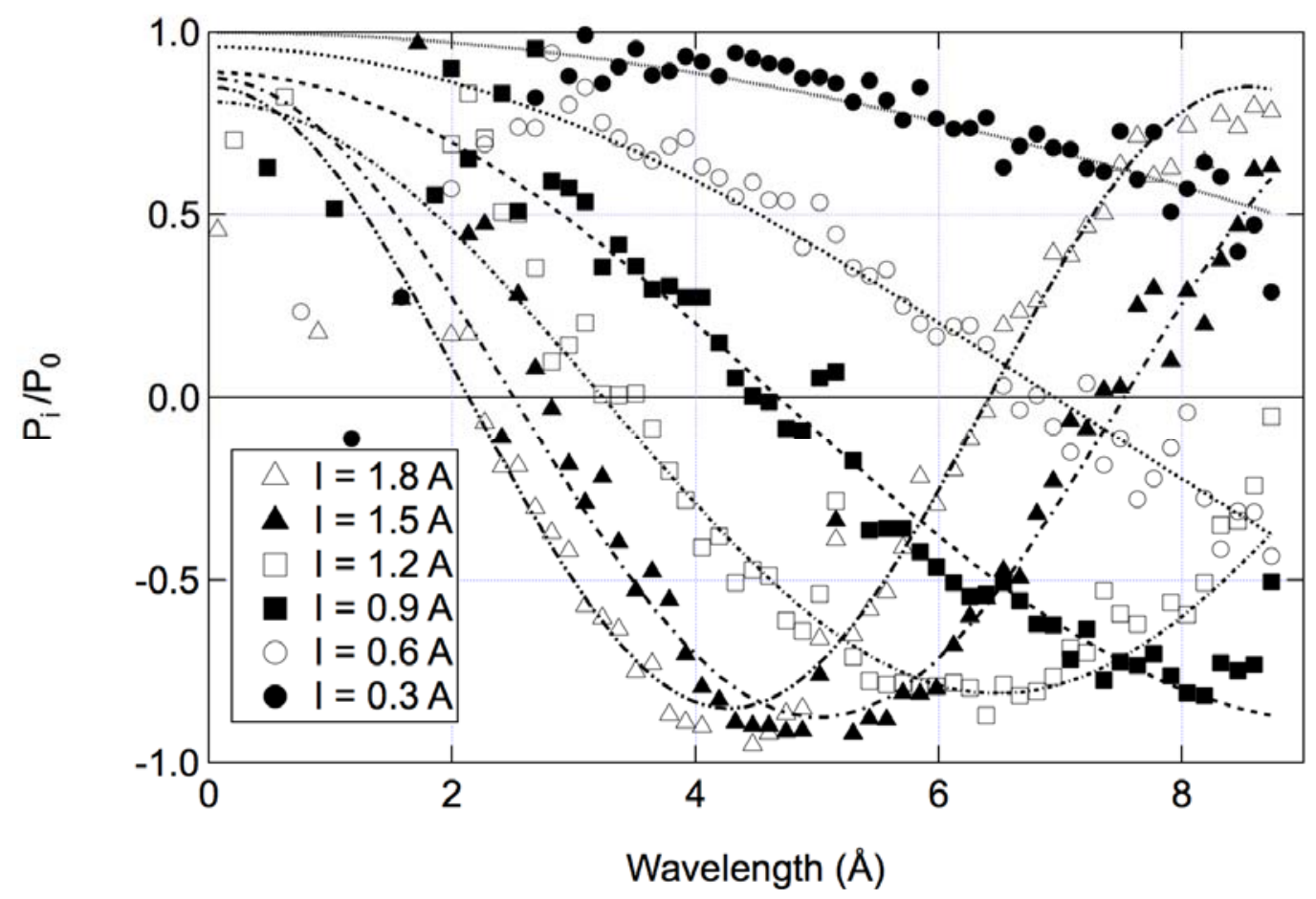


Figure-5 black and white
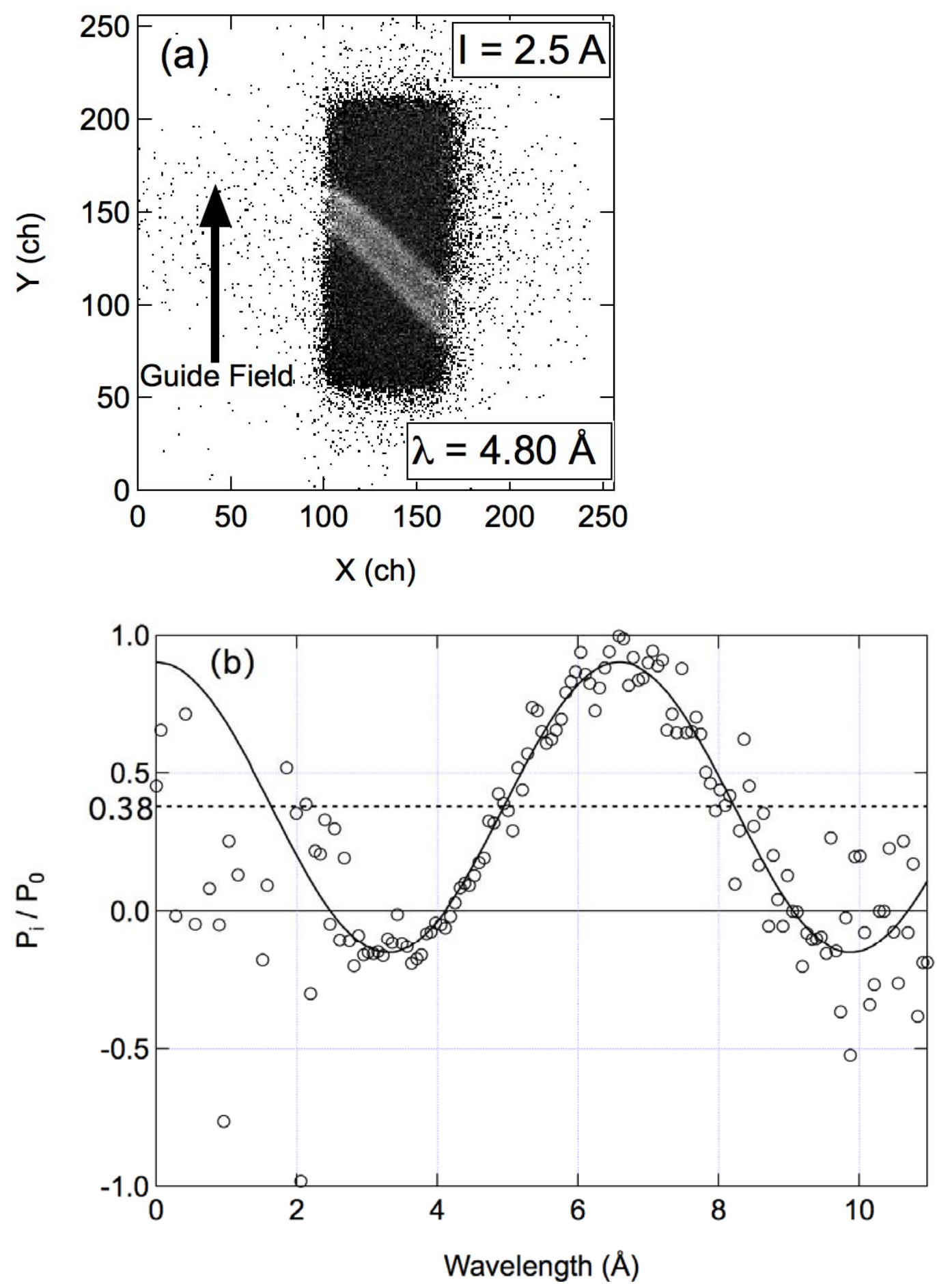